04

\title{
Электродинамический плазменный двигатель с капиллярно-пористыми электродами
}

\author{
(С) Ю.В. Мартыненко, ${ }^{1,2}$ М.Ю. Нагель ${ }^{1}$ \\ ${ }^{1}$ Национальный исследовательский центр „Курчатовский институт“, \\ 123182 Москва, Россия \\ ${ }^{2}$ Национальный исследовательский ядерный университет МИФИ, \\ 115409 Москва, Россия \\ e-mail: mifst04nmy@mail.ru, martyn907@yandex.ru
}

Поступило в Редакцию 11 февраля 2021 г.

В окончательной редакции 4 апреля 2021 г.

Принято к публикации 20 апреля 2021 г.

\begin{abstract}
Предложена схема двигателя для космических аппаратов на основе стационарного электродинамического плазменного ускорителя с капиллярно-пористыми электродами. Такие электроды возобновляемы и не деградируют, а срок их службы ограничен запасом металла-наполнителя. Найдены режимы, при которых капиллярно-пористые электроды эффективны. При этих режимах рассмотренный ускоритель может дать тягу более десяти ньютонов и удельный импульс десятки $\mathrm{km} / \mathrm{s}$. В качестве металла-наполнителя капиллярнопористых электродов могут быть использованы Li, S и Ga с пористыми матами из молибдена или вольфрама.
\end{abstract}

Ключевые слова: плазменный двигатель, тяга, удельный импульс, капиллярно-пористые электроды.

DOI: 10.21883/JTF.2021.09.51213.31-21

\section{Введение}

В настоящее время ведутся интенсивные работы по созданию мощных двигателей для космических аппаратов [1-4]. Важной характеристикой таких двигателей является долговечность. Одним из решений проблемы долговечности являются безэлектродные двигатели [5-7]. Другим решением может стать двигатель с возобновляемыми и недеградирующими электродами, например, плазменный двигатель с капиллярно-пористыми электродами [8]. Капиллярно-пористый электрод состоит из корпуса с расплавом металла (рис. 1,a). Его сторона, обращенная к плазме, состоит из пористых матов (рис. $1, b)$, пропитанных жидким металлом, поступающим за счет поверхностного натяжения, при этом корпус соединен с резервной емкостью с расплавом металла (рис. 1,a). Поверхность электродов, обращенная к плазме, все время покрыта слоем расплавленного металла, возобновляемого за счет капиллярных сил, и не деградирует.

Такие электроды возобновляемы, их срок службы ограничен запасом металла, используемого для расплава. Использование капиллярно-пористых электродов возможно в различных типах плазменных двигателей. В работе [8] предлагался магнитогидродинамический укоритель с капиллярно-пористыми электродами. Здесь рассмотрено электродинамическое ускорение плазмы, впервые предложенное в [9], в стационарном режиме работы плазменного ускорителя. В качестве плазмообразующего вещества выбран гелий. В настоящей работе определяются режимы, при которых капиллярнопористые электроды эффективны. Предлагается коаксиальный плазменный ускоритель, состоящий из внешнего электрода радиуса $R_{1}$ и внутреннего радиуса $R_{0}$ (рис. 2 ). Полезная длина электродов $l$. Ускоряемой массой является плазма, которая замыкает электрическую цепь и, находясь в магнитном поле тока в электродах, приобретает ускорение вследствие силы Ампера. В настоящей работе предлагается общая концепция двигателя на основе стационарного электродинамического плазменного ускорителя с капиллярно-пористыми электродами. Детальная разработка такого двигателя, изучение, например, таких вопросов, как неустойчивости плазмы в ускорительной камере, не входит в задачу настоящей работы.

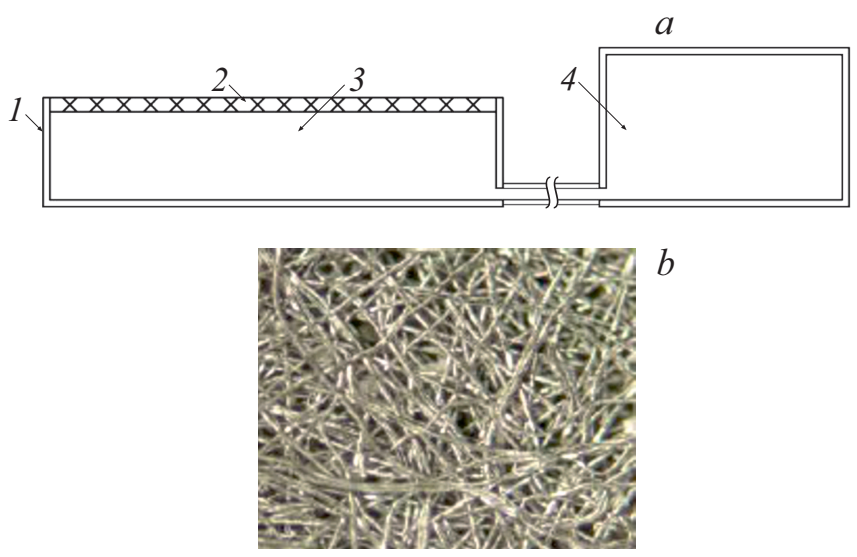

Рис. 1. $a-$ схема капиллярно-пористого электрода: корпус электрода 1 , сторона, обращенная к плазме пористого мата 2, заполненного жидким металлом и контактирующая с объемом жидкого металла 3 , пополняемого из резервного объема с жидким металлом $4 ; b-$ пористый мат. 


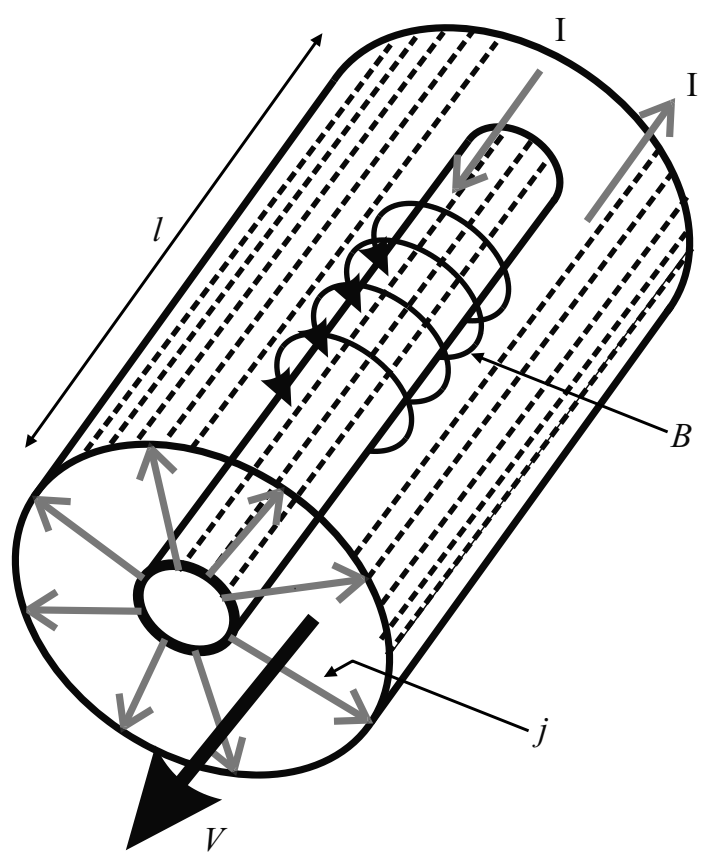

Рис. 2. Схема коаксиального плазменного электродинамического ускорителя.

\section{1. Стационарный режим плазменного ускорителя}

Полагается, что на входе в ускоритель гелий ионизуется, и образовавшаяся плазма потом ускоряется на длине $l$. Способ создания плазмы - высокочастотный или дуговой разряд - здесь не рассматривается. Мы рассматриваем только ускорение плазмы в аксиальном ускорителе с капиллярно-пористыми электродами.

Уравнения, описывающие ускорение плазмы в ускорителе, следующие. Неразрывность потока плазмы дает зависимость плотности плазмы $\rho=M \cdot n(M-$ масса атома Не, $n$ - число атомов Не в единице объема) от координаты $x$ вдоль ускорителя:

$$
\rho(x)=\rho_{0} V_{0} / V(x),
$$

где $\rho_{0}$ и $V_{0}$ - плотность плазмы и ее начальная скорость на входе в ускоритель, $V(x)$ - скорость плазмы на расстоянии $x$ от входа в ускоритель.

Сила, действующая на единицу объема плазмы в ускорителе, равна

$$
\rho(x) \frac{d V}{d t}=\langle j(x) B(x)\rangle,
$$

где $\langle j B\rangle-$ усредненное по сечению произведение плотности тока и магнитного поля, создаваемого током в электродах. Мы рассматриваем параметры плазмы с малым давлением и не учитываем градиент давления в (2). Сопротивление плазмы много больше сопротивления электродов. Обобщенный закон Ома

$$
j(x, r)=\sigma(E(r)-V(x) \cdot B(x, r)),
$$

где $\sigma$ - проводимость плазмы, $E=U / r \cdot \ln \left(R_{1} / R_{o}\right)-$ напряженность электрического поля в коаксиальном ускорителе, $U$ - разность потенциалов в плазме между электродами.

Проводимость плазмы перпендикулярно магнитному полю равна

$$
\sigma=\sigma_{0} /\left(1+\beta^{2}\right)
$$

где $\sigma_{0}=\left(e^{2} n / m\right) \tau-$ проводимость плазмы без магнитного поля, $e$ и $m-$ заряд и масса электрона, $v_{e}-$ тепловая скорость электронов, $\beta=\omega \tau$ - параметр Холла, $\omega=e B / m$ - циклотронная частота электрона, $\tau=\left(n \sigma_{e i} v_{e}\right)^{-1}$ и $\sigma_{e i}-$ время и сечение столкновения электрона с ионами. Нагрев плазмы быстро приводит к ее ионизации, так что можно не учитывать столкновения электронов с нейтральными атомами. При однократной ионизации $\sigma_{e i} \approx 10^{-16} / T^{2}\left(\mathrm{~m}^{2}\right)$, где $T-$ электронная температура в $\mathrm{eV}$ и $\sigma_{0} \approx 400 T e_{e}^{3 / 2}(\Omega \cdot \mathrm{m})^{-1}$.

Время передачи энергии от электронов к ионам $t=(M / m) \cdot \tau$ много меньше времени пролета ускорительной камеры $l / V \approx 10^{5}-3 \cdot 10^{-5}(\mathrm{~s})$. Потому температура $T_{i}$ ионов равна температуре электронов $T$, а ускорение, приобретаемое электронами под действием силы Ампера, передается ионам, т.е. плазма ускоряется как единое целое.

Время двукратной ионизации и время рекомбинации ионов Не+ много больше времени пролета ускорительной камеры при $n<10^{24}\left(\mathrm{~m}^{-3}\right)$, поэтому степень ионизации не меняется в процессе ускорения плазмы. Мы полагаем для простоты, что плазма, поступающая в ускоритель, однократно ионизована и степень ионизации $\alpha \approx 1$.

Температура электронов $T$ определяется из уравнения

$$
\begin{aligned}
3 n \cdot d T / d t & =\left\langle j^{2}\right\rangle / \sigma-W_{R}-q_{c} \cdot 2 R_{1} /\left(R_{1}^{2}-R_{o}^{2}\right) \\
& -q_{a} \cdot 2 R_{o} /\left(R_{1}^{2}-R_{o}^{2}\right),
\end{aligned}
$$

где $W_{R}$ - мощность излучения плазмы, последние два слагаемых в правой части - мощность, выделяемая на электродах (см. разд. 2), отнесенная к единице объема плазмы. Оценка на основе базы данных [10] показала, что $W_{R}$ мало, в расчетах оно не учитывалось. Плотность тока $j$ связана с током в электродах :

$$
j(x, r)=-d I / d x \cdot(1 / 2 \pi r)
$$

а магнитное поле равно

$$
B(x, r)=\frac{\mu_{0}}{4 \pi} \int_{x_{0}}^{l} \frac{r I\left(x_{1}\right) d x_{1}}{\left(\left(x-x_{1}\right)^{2}+r^{2}\right)^{3 / 2}} .
$$

Граничные условия

$$
\begin{gathered}
x=0: \quad \rho(0)=\rho_{0}, \quad V(0)=V_{0}, \quad T=T_{0}, \\
x=\ell: \quad I(\ell)=0, \quad V(\ell)=V,
\end{gathered}
$$

где $V_{0}$ - тепловая скорость, соответствующая начальной температуре плазмы $T_{0}, V-$ конечная скорость плазмы. Система уравнений (1)-(7) с условиями (8), (9) описывает ускорение плазмы. Для простоты все рассчитываемые величины усреднены по сечению ускорителя. 


\section{2. Капиллярно-пористые электроды}

Для определения условий работы капиллярнопористых электродов надо знать тепловую нагрузку на электроды. Определим потенциал плазмы по отношению к катоду $U_{c}$. В отсутствие эмиссии электронов с катода при $T_{i}=T_{e}=T$, т.е. при выполненном условии Бома, а также при $j<e n v_{i} / 4$ потенциал $U_{k}$ определяется из баланса потоков зарядов, обеспечивающего ток $j$ :

$$
\frac{1}{4} e n v_{i}-\frac{1}{4} e n v_{e} \cdot \exp \left(-e \cdot U_{c} / T\right)=j,
$$

где $v_{i}=(2 e T / M)^{1 / 2}, v_{e}=(2 e T / m)^{1 / 2}$. Тогда потенциал $U_{c}$ равен

$$
U_{c}=\frac{T}{e} \ln \left(\frac{v_{e}}{v_{i}-4 j / e n}\right) .
$$

Поток тепла на катод равен

$$
\begin{aligned}
q_{c} & =\left(e n v_{i} / 4\right) \cdot\left(U_{c}+\left(I_{1}-\varphi\right)+1.5 T\right) \\
& +\left(\left(v_{i}-4 j /(e \cdot n)\right) n / 4\right) \cdot 1.5 T
\end{aligned}
$$

где $\varphi$ - работа выхода. В (10) учитывается энергия нейтрализации ионов. Отраженные и десорбированные атомы гелия нейтральны.

Потенциал плазмы относительно анода $U_{a}$ устанавливается также из условия обеспечения тока с плотностью $j$ на анод. На анод поток ионов пренебрежимо мал, а поток электронов при $e n v_{e} / 4>j$ равен

$$
j=\frac{1}{4} n v_{e} \cdot \exp \left(e-U_{a} / T\right)
$$

Отсюда

$$
U_{a}=\frac{T}{e} \ln \left(\frac{e n v_{e}}{4 j}\right) .
$$

Поток тепла на анод

$$
q_{a}=j \cdot(1.5 \cdot T+\varphi) .
$$

В качестве внешнего электрода предлагается брать катод, обоснование приведено в разд. 3.

Металл наполнителя в капиллярно-пористой системе (КПС) испаряется под действием тепловой нагрузки. Капиллярно-пористые электроды не деградируют, но их испарение приводит к потере металла наполнителя. Для эффективности ускорителя с капиллярнопористыми электродами потеря массы материала наполнителя должна быть, по крайней мере, в $\gamma=10$ раз меньше потери массы гелия $\frac{d m_{\text {не }}}{d t}=\rho(0) \cdot V(0) \cdot S$, где $S=\pi \cdot\left(R_{1}^{2}-R_{0}^{2}\right)$. Величина $\gamma$ выбрана достаточно произвольно. Но температура поверхности и допустимая плотность мощности зависят от этой величины слабо (при изменении $\gamma$ в 2 раза допустимая температура меняется менее чем на $10 \%$, что видно из (12), (13)), поэтому этот выбор мало влияет на результат.
При отводе тепла от поверхности теплопроводностью температуру поверхности можно оценить как

$$
T=T_{c}+q_{(c, a)} d / K,
$$

где $d$ - расстояние от поверхности электрода до „холодильника“ с температурой $T_{c}$, которая должна быть выше температуры плавления металла наполнителя и по которому отводится тепло, $K-$ коэффициент теплопроводности. Скорость потери массы металла наполнителя КПС с единицы поверхности электрода равна

$$
\frac{d m}{d t}=\rho_{m} s \cdot \exp (-H / T)
$$

где $\rho_{m}-$ плотность металла-наполнителя, $s \cdot \exp (-H / T) \quad$ - скорость движения границы поверхности в результате испарения, $s=(3 / 4 \pi)^{1 / 2} \cdot c$ $c-$ скорость звука, $H-$ энергия испарения [11]. Полная скорость потери массы электрода равна интегралу выражения (12) по поверхности электрода и должна быть меньше $\frac{1}{\gamma} \frac{d m_{\text {He }}}{d t}$ :

$$
\frac{d M}{d t}=\int_{0}^{L} d m / d t \cdot 2 \pi R_{0,1} d x
$$

Мы пренебрегаем влиянием испаренного металла на параметры плазмы, поскольку доля металлических ионов в плазме мала при выполнении условия (13).

В качестве металла наполнителя КПС в работе [12] были рассмотрены литий, галлий и олово. Галлий и олово имеют большую энергию испарения и более выгодны с точки зрения скорости испарения (табл. 1). Однако при высоких температурах они растворяют другие металлы. Наиболее стойкие $\mathrm{W}$ и Мо могут работать в контакте с $\mathrm{Ga}$ и $\mathrm{Sn}$ при температуре $T<600^{\circ} \mathrm{C}$ [12], в то время как у $\mathrm{Li}$ предел совместимости составляет $1200^{\circ} \mathrm{C}$ для Мо и $1500^{\circ} \mathrm{C}$ для W. Поэтому для $\mathrm{Ga}$ и $\mathrm{Sn}$ ограничением использования является максимальная температура электродов и соответственно плотность мощности, рассчитываемая по формулам (10) и (11) (табл. 1).

Таблица 1. Сравнительные характеристики $\mathrm{Li}, \mathrm{Ga}$ и $\mathrm{Sn}$

\begin{tabular}{c|c|c|c}
\hline Характеристики & ${ }_{3} \mathrm{Li}^{7}$ & ${ }_{31} \mathrm{Ga}^{70}$ & ${ }_{505} \mathrm{Sn}^{119}$ \\
\hline$\rho, \mathrm{kg} / \mathrm{m}^{3}$ & 534 & 5900 & 7310 \\
\hline$T_{m}, \mathrm{~K}$ & 453 & 302 & 505 \\
\hline$H, \mathrm{eV}$ & 1.48 & 2.73 & 2.98 \\
\hline Теплопроводность, $\mathrm{W} /(\mathrm{m} \cdot \mathrm{K})$ & 47 & 27 & 32 \\
\hline$T_{\max }$ совместимости, ${ }^{\circ} \mathrm{C}$ & $\begin{array}{c}1200(\mathrm{Mo}), \\
1500(\mathrm{~W})\end{array}$ & $\begin{array}{c}600 \\
\mathrm{Mo}, \mathrm{W}\end{array}$ & $\begin{array}{c}600 \\
\mathrm{Mo}, \mathrm{W}\end{array}$ \\
\hline$\langle q\rangle_{\max }, \mathrm{MW} / \mathrm{m}^{2}$ & 1 & 1.5 & 1
\end{tabular}


Таблица 2. Значения скорости $V$, тяги $F$, расхода рабочего вещества $($ Не), цены тяги, мощности ускорителя, энергетической эффективности $\eta$ при разных значениях начальной плотности плазмы $n$, радиусов внутреннего $\left(R_{0}\right)$ и внешнего $\left(R_{1}\right)$ электродов, напряжения между электродами $U$

\begin{tabular}{c|c|c|c|c|c|c|c|c}
\hline$n, \mathrm{~m}^{-3}$ & $0.3 \cdot 10^{21}$ & $0.4 \cdot 10^{21}$ & $0.4 \cdot 10^{21}$ & $0.5 \cdot 10^{21}$ & $0.5 \cdot 10^{21}$ & $0.5 \cdot 10^{21}$ & $1.0 \cdot 10^{21}$ & ЭРД $[4]$ \\
\hline$R_{0}, \mathrm{~cm}$ & 2 & 2 & 3 & 2 & 2.5 & 3 & 2 & \\
\hline$R_{1}, \mathrm{~cm}$ & 10 & 9 & 10 & 9 & 8 & 7 & 8 & \\
\hline$U, \mathrm{~V}$ & 70 & 60 & 52 & 56 & 40 & 26 & 48 & \\
\hline$W, \mathrm{MW}$ & 1.0 & 1.0 & 1.0 & 1.0 & 0.7 & 0.5 & 1.0 & 0.5 \\
\hline$V, \mathrm{~km} / \mathrm{s}$ & 48 & 44 & 41 & 39 & 37 & 34 & 31 & 50 \\
\hline$F, \mathrm{~N}$ & 23 & 22 & 25 & 25 & 18 & 11 & 31 & 12 \\
\hline Цена тяги, kW/N & 43 & 45 & 40 & 40 & 39 & 45 & 32 & 83 \\
\hline$d m_{\text {не } / d t, \mathrm{~g} / \mathrm{s}}$ & 0.5 & 0.5 & 0.6 & 0.6 & 0.5 & 0.3 & 1.0 & 0.1 \\
\hline$\eta$ & 0.50 & 0.51 & 0.50 & 0.50 & 0.46 & 0.40 & 0.47 & 0.6
\end{tabular}

В работе [13] показано, что даже при скорости испарения $0.1 \mathrm{~g} /\left(\mathrm{cm}^{2} \cdot \mathrm{s}\right)$ капиллярные силы успевают восстанавливать слой лития на поверхности. Капиллярные силы $\mathrm{Sn}$ и $\mathrm{Ga}$ также позволяют использовать их в качестве капиллярно-пористых электродов [12]. Так что все рассмотренные металлы $\mathrm{Li}, \mathrm{S}$ и $\mathrm{Ga}$ можно использовать в качестве металла-наполнителя с пористыми матами из молибдена или вольфрама.

\section{3. Результаты расчетов стационарного плазменного ускорителя}

Система уравнений (1)-(7) граничными условиями (8) и (9) решалась численно при различных значениях плотности плазмы $n$ и радиусах электродов $R_{0}, R_{1}$. Температура плазмы на входе в ускоритель была $1.6 \mathrm{eV}$.

Падение напряжения в плазме $U$ подбиралось так, чтобы удовлетворить следующим ограничениям:

1) полная мощность не более $1 \mathrm{MW}$ (были рассмотрены значения 0.5, 0.7 и $1 \mathrm{MW}$ ),

2) скорость потери массы металла наполнителя в $\gamma$ раз меньше скорости потери массы Не (см. (13)),

3) концентрация плазмы ограничена снизу $\left(n>3 \cdot 10^{20} \mathrm{~m}^{-3}\right) \quad$ условием $\quad \beta<1 \quad$ (при $\beta>1$ проводимость плазмы убывает с ростом $\beta$ ), ограничения сверху нет, но при росте концентрации падает скорость плазмы и энергетическая эффективность.

Длина ускорителя выбрана из следующих соображений. В средней части камеры ускорение практически отсутствует. Поэтому нерационально выбирать слишком большое $\ell$. При слишком малом $\ell$ области ускорения вначале и в конце камеры перекрываются, и общее ускорение уменьшается. Наиболее оптимальная длина ускорителя $\ell=0.4 \mathrm{~m}$.
Результаты расчетов приведены в табл. 2. Энергетическая эффективность двигателя $\eta$ оценивалась как

$$
\eta=W_{u} /\left(U_{b} \cdot I_{b}+W_{i}\right)
$$

где $W_{u}=\rho_{0} \cdot V_{0} \cdot S V^{2}(l) / 2$ - полезная мощность; $U_{b}$ напряжение на батарее, оно складывается из напряжения $U$, определяющего ток в плазме, и разности напряжений в приэлектродных слоях; $W_{i}$ - мощность, необходимая для начального нагрева и ионизации плазмы:

$W_{i}=\left(3 T_{0}+I\right) \cdot n_{0} V_{0} S$, где $T_{0}$ - начальная температура плазмы. Эта оценка $\eta$ включает основные каналы расхода энергии, но не учитывает менее энергетически емкие каналы потребления энергии, такие, как поддержание необходимой температуры капиллярнопористых электродов, подача гелия и прочее. Сравнение энергетической эффективности, рассчитанной нами, и $\eta$ магнитоплазмодинамического двигателя [1] с таким же принципом ускорения плазмы, показывают, что неучтенные нами затраты энергии на обеспечение работы ускорителя не превышают нескольких процентов от полного расхода энергии. А затраты на поддержание необходимой температуры капиллярно-пористых электродов, хотя и зависят сильно от конструкции системы, но по опыту авторов работы [13] не превышают $\sim 1 \mathrm{~kW}$.

Приведем формулы для других важнейших характеристик двигателя.

Тяга

$$
F=\rho_{0} V_{0} \cdot S \cdot V(l) .
$$

Расход рабочего вещества (Не) составляет

$$
d m_{\mathrm{He}} / d t=\rho_{0} V_{0} \cdot S .
$$

Отметим, что испарение $\mathrm{Li}$ растет при увеличении плотности тока $j$ вследствие увеличения тепловой нагрузки на начальном и конечном участках ускорения, 


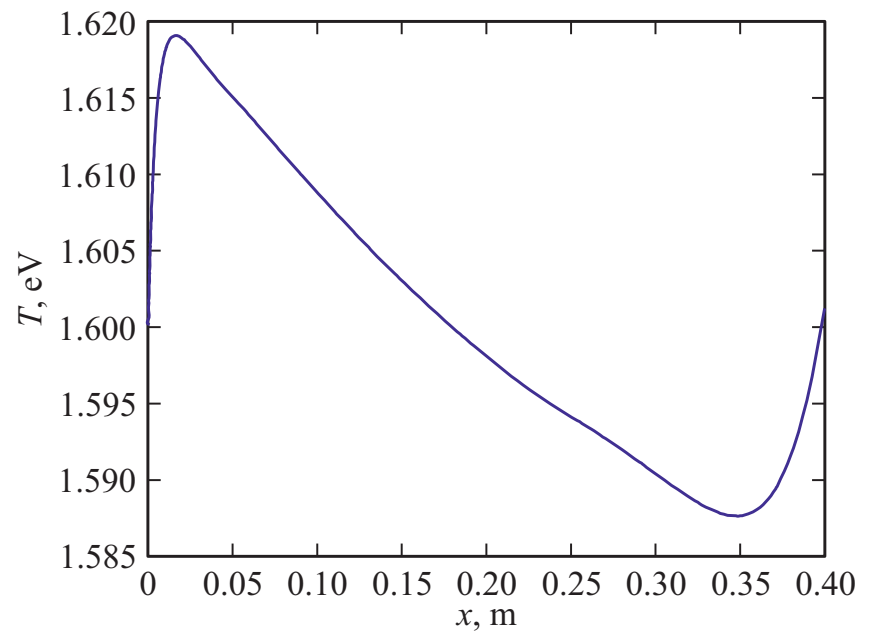

Рис. 3. Температура плазмы как функция расстояния от начала ускорения при $R_{0}=2 \mathrm{~cm}, R_{1}=10 \mathrm{~cm}, n=3 \cdot 10^{20} \mathrm{~m}^{-3}$.

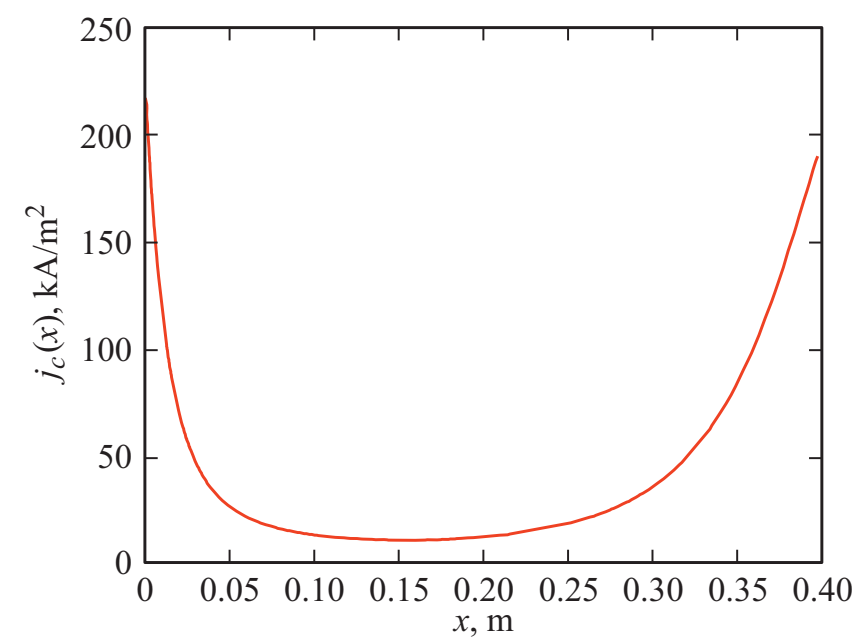

Рис. 4. Плотность тока на катод как функция расстояния $x$ от начала ускорения при $R_{0}=2 \mathrm{~cm}, R_{1}=10 \mathrm{~cm}, n=3 \cdot 10^{20} \mathrm{~m}^{-3}$.

которые дают основной вклад в испарение Li. Ограничение плотности тока означает ограничение скорости истечения плазмы $V$. Поэтому ограничение (13) означает ограничение скорости $V$. Также отметим, что энергетическая эффективность $\eta$ растет с ростом мощности и при понижении плотности плазмы.

Зависимости температуры плазмы, плотности тока на катод и скорости от координаты приведены на рис. 3-5. Видно, что основную роль в ускорении плазмы играют начальный и конечный участки. По этой же причине электроды на этих участках испытывают наибольшую тепловую нагрузку (рис. 6). Также из рис. 6 видно, что тепловая нагрузка на катод значительно больше, чем на анод, даже если последний используется в качестве внутреннего электрода (с большей плотностью тока $j$ ). Поэтому именно условия на катоде определяют допустимые режимы работы ускорителя с капиллярнопористыми электродами.
На всем участке ускорения температура плазмы меняется в пределах $\sim 3 \%$. Небольшой рост температуры (на $1.5 \%$ ) происходит на участках наибольшего ускорения, в

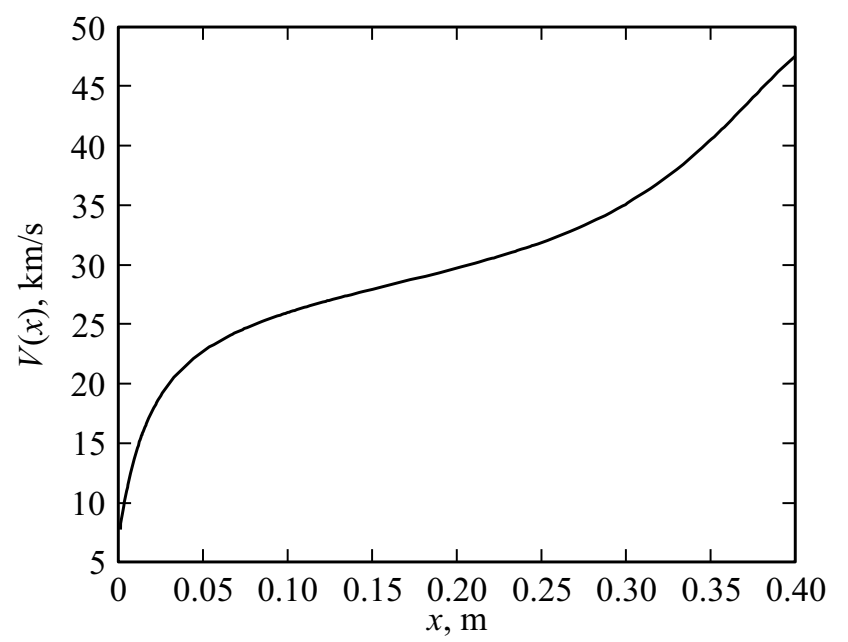

Рис. 5. Скорость плазмы как функция расстояния от начала ускорения при $R_{0}=2 \mathrm{~cm}, R_{1}=10 \mathrm{~cm}, n=3 \cdot 10^{20} \mathrm{~m}^{-3}$.
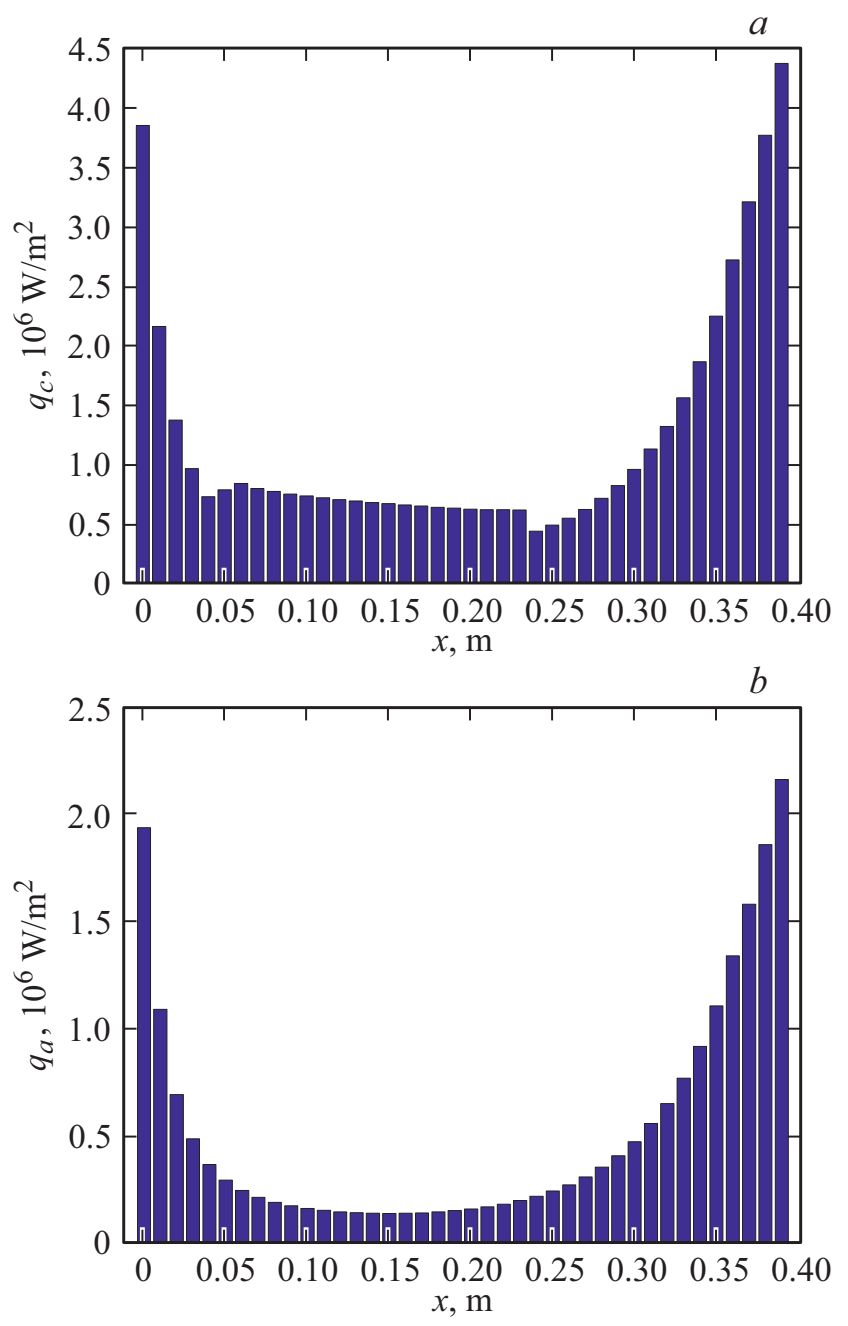

Рис. 6. Тепловая нагрузка на катод $(a)$ и анод $(b)$ при $R_{0}=2 \mathrm{~cm}, R_{1}=10 \mathrm{~cm}, n=3 \cdot 10^{20} \mathrm{~m}^{-3}$ в зависимости от $x$. 
начале и в конце ускорения, в средней части камеры ускорения происходит небольшое снижение $T$ (на 3\%) вследствие охлаждения на электродах.

Для уменьшения нагрузки на начальном участке ускорения можно предложить предварительное ускорение плазмы с меньшей напряженностью электрического поля. Для конечного участка ускорения можно предложить схему, допускающую $I(l) \neq 0$, т. е. дополнительное замыкание концов электродов.

Для сравнения в табл. 2 приведены параметры одного из наиболее продвинутых двигателей [4].

\section{Заключение}

Рассчитаны режимы электродинамического плазменного двигателя для космических аппаратов с капиллярно-пористыми электродами, при которых такие электроды эффективны. Такие электроды возобновляемы и не деградируют, а срок их службы ограничен запасом металла-наполнителя капиллярно-пористого электрода. Однако для эффективности двигателя необходимо ограничение температуры электродов. В случае Ga и Sn в качестве металла-наполнителя ограничение температуры диктуется совместимостью этих металлов с металлами, из которых изготовлены маты (Мо и W). Допустимые режимы ускорителя с использованием капиллярнопористых электродов позволяют при тяге $F$ более 10 ньютонов иметь удельный импульс $V$ десятки $\mathrm{km} / \mathrm{s}$.

\section{Благодарности}

Авторы благодарны М.Н. Казееву за полезные обсуждения.

\section{Конфликт интересов}

Авторы заявляют, что у них нет конфликта интересов.

\section{Список литературы}

[1] A.S. Voronov, A.A. Troitskiy, I.D. Egorov, S.V. Samoilenkov, A.P. Vavilov. J. Phys.: Conf. Series, 1686, 012023 (2020). DOI: $10.1088 / 1742-6596 / 1686 / 1 / 01202$

[2] И.А. Хмелевской, Д.А. Томилин. ЖТФ, 89 (9), 1360 (2019). DOI: 10.21883/JTF.2021.09.51213.31-21

[I.A. Khmelevskoi, D.A. Tomilin. Tech. Phys., 64 (9), 1283 (2019). DOI: 10.1134/S1063784219090068]

[3] I.V. Romadanov, A.I. Smolyakov, Y. Raitses, I. Kaganovich, T. Tian, S. Ryzhkov. Phys. Plasmas, 23 (12), 122111 (2016). DOI: $10.1063 / 1.4971816$

[4] В.П. Агеев, В.Г. Островский. Известия АН. Энергетика, 3, 83 (2007)

[5] S.N. Bathgate, M.M. Bilek., D.R. McKenzie. Plasma Sci. Technol., 19 (8), 3001 (2017).

DOI: $10.1088 / 2058-6272 /$ aa71fe

[6] М.В. Ковальчук, В.И. Ильгисонис, В.М. Кулыгин. Природа, 12, 33 (2017).
[7] В.А. Жильцов, В.М. Кулыгин. ВАНТ, сер. термоядерный синтез, 41 (3), 21 (2018).

[8] В.И. Ильгисонис, Ю.В. Мартыненко. Физика плазмы, 45 (1), 63 (2019). DOI: 10. 1134/S0367292119010062 [V.I. Ilgisonis, Yu.V. Martynenko. Plasma Phys. Reports, 45, 57 (2019). DOI: 10.1134/s1063780x19010069]

[9] Л.А. Арцимович, С.Ю. Лукьянов, И.М. Подгорный, С.А. Чуватин. ЖЭТФ, 33, 3 (1958).

[10] ADAC http://open.adas.ac.uk/detail/adf11/plt42/plt42_ar.dat

[11] С.И. Анисимов, Я.А. Имас, Г.С. Романов. Действие излучения большой мощности на металлы (Наука, М., 1970), c. 100 .

[12] И Е. Люблинский, А.В. Вертков, В.В. Семенов. ВАНТ, сер. термоядерный синтез, 38 (1), 7 (2015).

[13] В.Б. Петров, Б.И. Хрипунов, В.В. Шапкин, Н.В. Антонов. BАНТ, сер. термоядерный синтез, 4, 23 (2004). 